\title{
Mutagenesis and Temperature-Sensitive Little Machines
}

\author{
María Pertusa, Hans Moldenhauer, Sebastián Brauchi, \\ Ramón Latorre, Rodolfo Madrid and Patricio Orio
}

Additional information is available at the end of the chapter

http://dx.doi.org/10.5772/50333

\section{Introduction}

In mammals a class of ion channels able to sense a wide range of temperatures $\left(0-60{ }^{\circ} \mathrm{C}\right)$ has evolved. These molecular thermodynamic machines called thermo Transient Receptor Potential (thermoTRP) are spread through the different TRP channel subfamilies having members inside the TRPM (melastatin) subfamily, where TRPM2, TRPM3, TRPM4 and TRPM5 are heat-activated, whereas TRPM8 is activated by cold. The TRPV (vanilloid) subfamily contains four thermoTRP channels (TRPV1, TRPV2, TRPV3 and TRPV4), which are all activated by heat; and TRPA1 (ankyrin) channel which is activated by noxious cold (reviewed in [28, 107], Figure 1). More recently, a member of TRPC (canonical) subfamily, TRPC5, was identified as a cold receptor in the temperature range $37-25^{\circ} \mathrm{C}$ [1].

Located in cutaneous nerve endings of thermoreceptors and nociceptors, and because extreme temperatures produce discomfort and pain, thermoTRP channels are involved in nociception and can be activated by a long list of other noxious stimuli such as low $\mathrm{pH}$ and irritant chemicals [2].

What characterizes these channels is their exquisite temperature sensitivity. Thermodynamic analyses reveal that thermoTRP channels undergo large enthalpy changes $(\Delta H)$ that account for their high temperature sensitivity [3-8]. For example, the enthalpy change between close and open in TRPV1 and TRPM8 involves $\Delta H s$ of $\sim 100 \mathrm{kcal} / \mathrm{mol}$ and $60 \mathrm{kcal} / \mathrm{mol}$, respectively [3,5]. It is obvious that in order to make the closed-open reaction reversible these enthalpy changes must be accompanied by large entropy $(\Delta S)$ changes. These activation enthalpies are 3-5 times the enthalpy change for voltage- or liganddependent channel gating ( $\Delta H \sim 20 \mathrm{kcal} / \mathrm{mol}$; [108]). Actually, Yao et al. [7] pointed out that in the case of TRPV1, the $\Delta H$ involved in the closed-open transition is equivalent to an electrical energy moving 71 unit charges across $60 \mathrm{mV}$ ! 


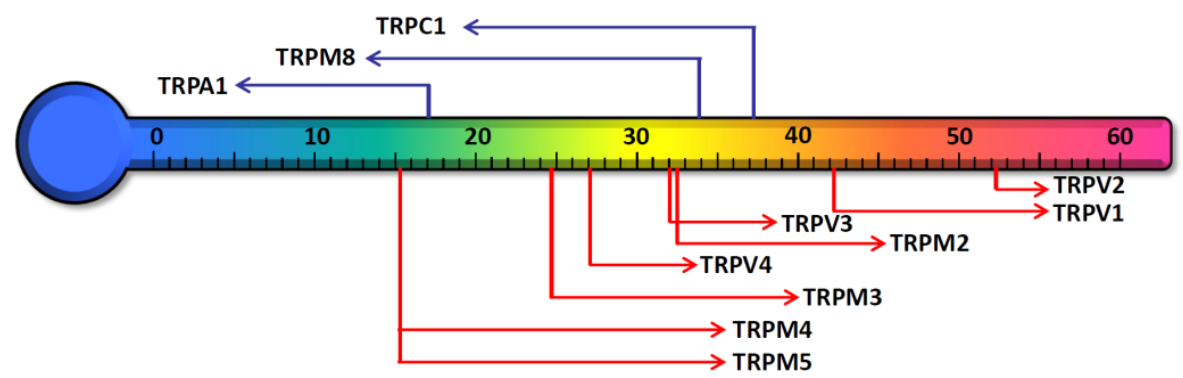

Figure 1. Schematic representation of the eleven mammalian thermoTRPs indicating their reported temperature sensitivity.

Enthalpy changes of this magnitude mean large structural rearrangements of the channelforming protein. Just consider that the $\Delta H$ of the thermal denaturation of the ribonuclease (RNase A), a 124 residues protein is $57 \mathrm{kcal} / \mathrm{mol}$ [9]. Since protein denaturation involves a change from a molecule with a very well defined structure to a random coil, how is it possible that that the TRPV1 channel opening reaction is defined by such a huge enthalpy change leading to $\mathrm{Q}_{10} \sim 270$ for TRPV1? It is difficult to think that, as in the process of denaturation, these channels undergo global disturbance of their structure, in particular given that channel opening demands a well kept pore structure and responses to voltage and agonists are maintained at all temperatures.

We rather are of the opinion that thermoTRPs might possess specific residues, domains or domain interactions that are specifically affected by temperature in the channel activation pathway. The data available at present strongly suggests that these structure(s) are different from those in charge of determining voltage-dependence or agonist binding [4].

The first goal of this chapter is to present the reader the different genetic and mutagenesis procedures that have been used so far in the quest for finding the "Holy Grail" of thermoTRP channels: the thermal sensor. The situation is at present rather confuse since the molecular determinants for temperature sensitivity in thermoTRP channels have been claimed to be in the N-terminus of TRPV1 and Drosophila TRPA1 [10,11], the pore region of TRPV1 and TRPV3 $[12,13]$ and in the C-terminus [14-16]. Bona fide components of such a thermal sensor can only be, however, those components capable of appreciably perturbing the enthalpy of the channel. Mutagenesis, as we describe below, has been indispensable in the search for those components.

In this chapter we will also show an analysis of the phenotypes of knockout mice that have been used so far in the study of the physiological role of these exciting temperature-sensitive little machines. We will see that several thermoTRPs are critical molecular components of the thermotransduction machinery in primary sensory neurons of the somatosensory system. We will also see that, in spite of their high temperature dependency, some thermoTRPs are playing roles apparently unrelated to their temperature sensibility. Thus, knockout mice have been of great value in unveiling both expected and unexpected roles for thermo TRP channels. 


\section{A chimeric approach to search for thermal sensors in thermoTRP channels}

Channels are most likely built as modular structures [4, 17-22], and we can hypothesize that temperature sensors need to be contained in these protein modules. The tale about the search for the temperature sensor in thermoTRP channels started with a deletion mutagenesis strategy designed to chop parts of the C-terminal domain. Such approximation renders phenotypes with altered thermal sensitivity where progressive deletions correlate with progressive loss of temperature dependency [15]. Prompted by the work of Vlachova et al [15], the group of Latorre engineered chimeras in which the entire C-terminal domains were swapped between cold (TRPM8) and hot receptors (TRPV1) [16]. The resultant chimeric channels -specially the one carrying TRPM8 C-terminal- inherits the temperature sensitivity of the channel contributing with the C-terminal, however, eliciting a rather small Q10 compared with WT channels. Interestingly, the chimeras were often unable to recover from either activation or deactivation process, this was described as "locking" behavior; extreme voltages or long incubations at different temperatures were needed to recover from that new state. The observations obtained on those chimeric proteins led to the conclusion that the C-terminal has an essential role as a "thermal modulator" of channel gating. This is not surprising considering the proximity of the swapped region to both the bundle crossing [23] and to the $\mathrm{PIP}_{2}$ regulatory region [24]. Further work on the C-terminal domain unveiled a short region in the C-terminal of TRPV1 able to change the TRPM8 phenotype to that of a heat receptor [14]. Although the studies described above did not unequivocally identify the C-terminal domain as the temperature sensor, these results demonstrated that the $\mathrm{PIP}_{2-}$, voltage- and thermal-responsive elements are contained in different channel molecular structures [14-16].

Recently, fluorescence resonance energy transfer (FRET) experiments done at the turret, a loop connecting the $5^{\text {th }}$ transmembrane domain with the pore helix and located above the external mouth of the pore, was reported to be involved in temperature sensing. Using FRET in combination with electrophysiological recordings and site directed mutagenesis, Yang et al. [25] showed that conformational rearrangements of the turret are essential for temperature-dependent activation. This result was somewhat supported by results presented by other groups in which pore mutations near the turret region either ablate or severely affect temperature-dependent gating [see high throughput section]. However, the striking results presented by Yang et al. have been severely questioned by the group of Qin who showed that deletions of the entire turret region are not affecting temperature sensitivity. Clearly this controversy has to be solved [26, 27].

This saga continues with a nice blend of the use of an ingenious fast temperature clamp $\left(>10^{5}{ }^{\circ} \mathrm{C} / \mathrm{s}\right)$ developed by Qin's laboratory [7], and mutagenesis to unveil structural domains in thermo-TRP channels that confer to these channels their exquisite temperature sensitivity. In this case, temperature jumps were produced using a single emitter laser diode as the heat source. This temperature clamp is able to change the temperature of the bath much faster than the time course of the development of the thermoTRP-induced currents (Figure 2). The 
technique allowed measuring directly activation and deactivation kinetics of the TRPV1 channel, a thermoTRP channel of the vanilloid family (Reviewed in [28]), as a function of temperature. The results indicated that the reaction path is asymmetrical, with temperature mainly driving the opening reaction while the closing rate is, if anything, sensitive to cold [7].

A

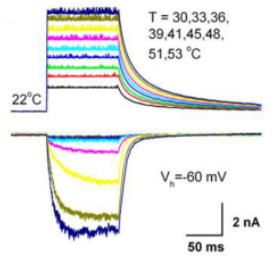

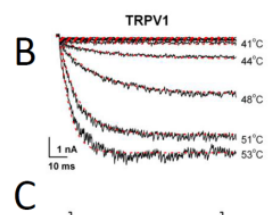

C

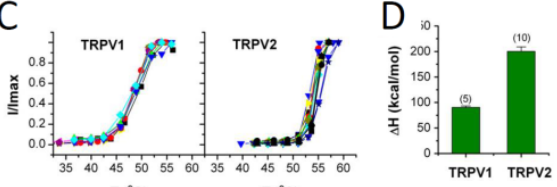

$\mathrm{E}$

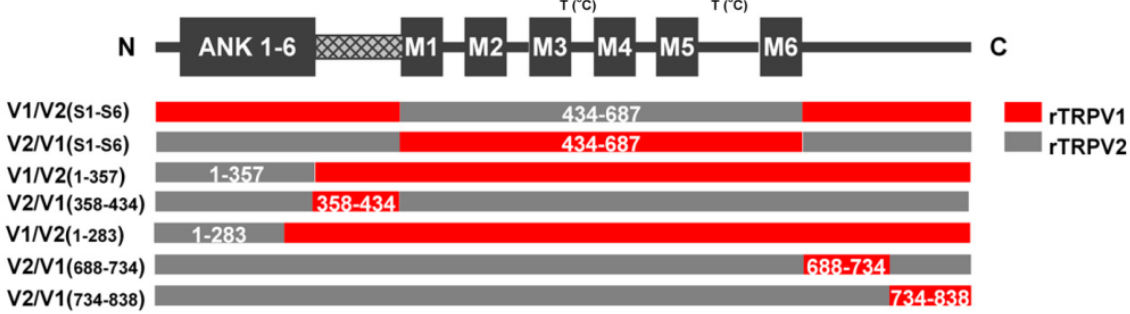

$\mathrm{F}$

V1/V2(S1-S6)
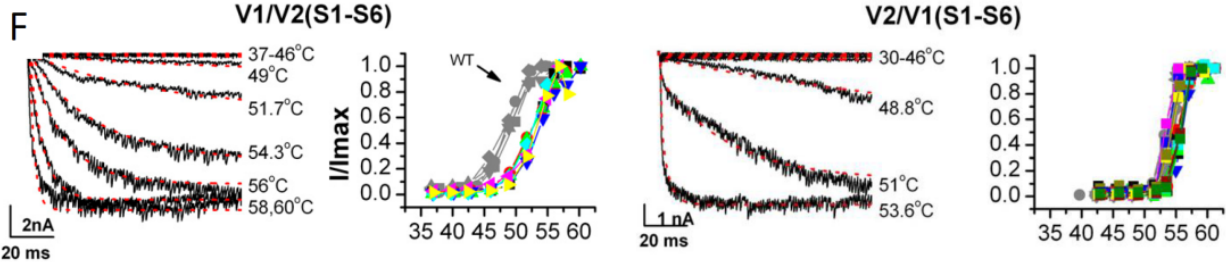

Figure 2. Searching for the molecular determinants of temperature sensitivity in thermoTRPV channels. A. Top. Submillisecond temperature steps generated by infrared laser irradiation. Bottom. TRPV1 channel responses induced by the rapid temperature changes shown in A. B. Comparison of the current time course of the TRPV1 currents (left) and TRPV2 (right). C. Temperature dependence of the steady state response taken from eperiments like those shown in B. D. Activation enthalpies for TRPV1 and TRPV2. E. Composition of the chimeric channel proteins used by Yao et al. [13]. F. Temperature sensitivity of TRPV1 and TRPV2 channels resides in the N-terminal. Notice that the V1/V2 chimera has a TRPV1 phenotype regarding gating kinetics and enthalpy changes, and that the V2/V1 chimera possesses a TRPV2 phenotype.

In a search for the protein domain(s) involved in thermal sensitivity of TRPV1 channels, Yao et al. [11] used TRPV1 that has an enthalpy change $(\Delta \mathrm{H})$ of activation of $\sim 100 \mathrm{kcal} / \mathrm{mol}$, and TRPV2 in which the channel closed-opening reaction involve a $\Delta H \sim 200 \mathrm{kcal} / \mathrm{mol}$. A systematic chimeric analysis on TRPV1 and TRPV2 allowed to conclude that temperature sensitivity is associated with the $\mathrm{N}$ terminus (Figure 2). In the TRPV1 N-terminus, they 
further identified $\mathrm{N}$-terminus a fragment of 80 residues that connects the ankyrin repeats to the first transmembrane segment able to transfer to TRPV2 the temperature sensitivity characteristics of TRPV1. Notably, this channel region is precisely the segment missing in the nonfunctional TRPV1b splice variant $[29,30]$. Alterations of this region profoundly altered the energetic of thermal sensing in all temperature-sensitive vanilloid receptor homologues (TRPV1-4), while its replacement in temperature-insensitive homologues successfully conferred gain-of-function. It is important to note here that swapping other domains like the $\mathrm{C}$ terminus or other domains did not have any effect on the temperature sensitivity. For example, a mutant containing the first 357 amino acids of TRPV2 (i.e., all 6 transmembrane domains and the $C$ terminus belonged to TRPV1) has a TRPV2 phenotype. These results demonstrated that these channels possess localized molecular components for temperature detection.

A chimeric strategy was also used to unveil the thermal-sensing structures in the TRPA1 channels. In this case, the group of David Julius took advantage of the fact that mammalian TRPA1 channels are heat insensitive while the snake TRPA1 version is activated by heat. Through engineering chimeras between mammalian and snake ion channels, the authors turned the mammalian TRPA1 channel into a temperature-sensitive channel identifying the N-terminal region of TRPA1 -within the ankyrin domain of the snake channel- that behave as transferable temperature sensitive modules. The chimeric approximation also suggests that both, sensitivity to chemical stimuli and intracellular calcium dependence, also localize to the N-terminal ankyrin repeat-rich domain [10].

Thus, the current scenario presents the N-terminal region of TRPV1, TRPV2 and TRPA1 as a strong candidate for containing the temperature-sensitive domain with the C-terminal playing a modulatory role. One interesting possibility envisioned by Brauchi et al. [16] was that temperature may affect the interaction between a particular portion of the proximal Cterminal and some other regions of the channel. It may be that the structural arrangements induced by temperature involve an inter-molecular interaction between the proximal Cterminal and specific regions of the N-terminal domain (eg. ANK domains in TRPVs). Such setting would be extremely convenient, because it could explain the large entropy associated to the hydrophobic effect $[3,31]$ without the necessity of an argument that involves protein unfolding. Lacking ANK domains, this hypothesis plotted for TRPVs and TRPA1 may not be necessarily valid for the case of TRPMs.

\section{High throughput mutagenesis and thermoTRP channels}

In the field of ion channels the mutagenesis is one of the most powerful tools to understand structural-function relationships. The most common strategy is the replacement of certain residue that one might consider important for channel function by another, and then to perform functional experiments to test our hypothesis of how a particular ion channel gates or transport ions. Depending on primer design you can even to replace 2 or 3 amino acids in a single PCR reaction but what can you do if the protein under study has at least 1000 amino acids? Repeating the single point mutation it is not an option if you must replace a large 
number of residues by one of the 20 amino acids. The picture becomes more complicated if you wish to test more than one amino acid at each position. This can be an incredible time (and money!) consuming task.

The ideal experimental maneuver would be to perform multiple point mutations of our protein of interest, by means of a technique that would allow you to obtain, for example, 12000 single-point mutations and to test the functional properties of each one of them in one day. Now the good news: that technique exists and is called high throughput mutagenesis coupled to a cell based assay using a Fluorescent Imaging Plate Reader (FLIPR).

The basis of this technique is a massive random mutagenesis in our target cDNA sequence. This is done by means of a PCR reaction using the blend of 2 specific error prone DNA polymerases, and depending on the commercial kit of choice the names could be Mutazyme I and Taq DNA polymerase mutant. The explanation for using a mix of enzymes is that we need the same frequency of mutations in the 4 nucleotides. In the past, the kits used only one enzyme, Mutazymes I and the frequency of mutation in the Cs and Gs was higher than in the As and Ts. The procedure starts by setting up how many mutations the DNA polymerase will introduce per clone, namely the mutation frequency. The whole idea is to obtain 1 mutation per clone, and this can be controlled by using the adequate amount of target DNA and number of PCR reaction cycles. High amounts of DNA give lower frequency of mutations, because one single molecule of DNA has less replication cycles; the same occurs with the PCR cycles in which high amount of DNA and less cycles give a lower mutation frequency.

This procedure yields, however, mutants clones with 2 and 3 mutations, but if those clones present an interesting phenotype we can design the single point mutants to evaluate the contribution of each mutation. Once the random mutagenesis process is finished you are left with a large library with thousand of mutants, with the only caveat that it is probable that you do not have every possible amino acid replacement in each position of a particular region of your protein. If certain mutations were particularly interesting after functional evaluation and are grouped in a well defined region, a second screening can be done, but this time in a saturating way, that means changing every amino acid by one of the other 20 . The procedure is the same describe above using the same mutagenesis approach, but with the difference that here we take a particular section of the protein primary structure and change every residue by each of the other 20 . To obtain this, it is necessary to take lower concentrations of DNA and to do more than 25 cycles of PCR (frequently 30). Often, to obtain a frequency equal or greater than 20 mutations per $\mathrm{Kb}$, it is necessary to perform many PCR reactions in tandem.

At this point, you have at least 10000 different mutants of your favorite protein, but this is completely useless unless you have a fast method to evaluate the functional properties of each one of them. This is done using the fluorimetric cell-based assay method dubbed FLIPR [32]. The machine is basically a fluorescent plate reader that can stimulate the sample in a specific wavelength and detect the change in the fluorescent emission in other wavelength (this wavelength depends on the fluorescent probe used). A remarkable advantage of the 
method is that it allows the use of 384-wells plate which makes easy to test the effect of chemical compounds on ion channel function, i.e. an agonist or a blocker. This method makes possible to perform a large number of experiments in a short period of time.

The 384-wells plate have a standardized quantity of mammalian cells like HEK-293 or CHO transfected with the ion channel DNA and each well contains a different mutant clone. The cells in every well are loaded with a fluorescent probe that can report the activity of our ion channel. If you are interested in studying ion channels one of the most important requirements for using this technique is that the channel allows the passage of ions that can be detected by a probe. For example, if you are working with a TRP channel that permeates $\mathrm{Ca}^{2+}$ we can use a calcium probe like Fluo-3 or Fluo-4. The technique is particularly suited for thermoTRP since these channels are polymodal receptors that are activated by different agonists and temperature and many of them are $\mathrm{Ca}^{2+}$ permeable $[12,13,33]$.

This is an unbiased approach, different from making chimeras between different channelforming proteins and other kind of mutations techniques. Since the mutations are done randomly, the high throughput mutagenesis technique produced mutants that are not biased by our previous knowledge of how ion channels may work. The discoveries using this approach in the field of thermoTRP channels are: a) important amino acid residues involved in temperature sensing; and b) the binding sites for the agonist menthol in mTRPM8 [12, 13, 33] (Figure 3).

For TRPV1, Grandl et al. [13] focused in a mutant library of 4400 clones from which they found 3 mutations that affect the heat response; N628K located in the pore region, adjacent to the pore helix and N652T and Y653T placed in the extracellular loop between the selectivity filter and transmembrane domain 6 (TM6). The three mutations show a decrease in their heat sensitivity as determined by a right shift in the temperature threshold to higher temperatures. The double and triple mutants N652T;Y653T and N628K;N652T; Y653T, have a stronger phenotype than the single-point mutants, with a greater decrease in their heat sensitivity. However, their activation by the agonists capsaicin and 2-Aminoethoxydiphenyl borate (2APB [34, 35]), as well as by voltage and acid $\mathrm{pH}$ is the same as in the wild type. As a quality control of the screening, they found 2 residues previously reported: E600V that produces a loss in the $\mathrm{pH}$ sensitivity [13] and F489Y that produces a right shift in the EC50 capsaicin activation. This kind of information gives us the confidence that the procedure is trustable since it corroborates the results obtained with other techniques.

In the case of mTRPV3, from a mutant library of 14.000 clones Grandl et al. [12] discovered 3 mutants with a decrease in their heat sensitivity; I644S, N647Y and Y661C all of them located between the pore helix and TM6. The 3 mutants have a normal response to the agonist $2 \mathrm{APB}$, and unaltered ion selectivity. This is important because the mutations are located near to the TRPV3 selectivity filter. These libraries are not saturating (i.e., every amino acid of the channel-forming protein replaced by one of the other 20), for this reason, Grandl et al. [12] made 45 more mutants in the region between TM5 and TM6 finding two other clones in TM6 with an altered temperature phenotype (F654S and L657E). Molecular modeling suggests that the three mutations in TM6 (F654S, L657E and Y661C) are located in a periodic pattern probably aligned on the lipid-facing side of the $\alpha$-helix[12]. 

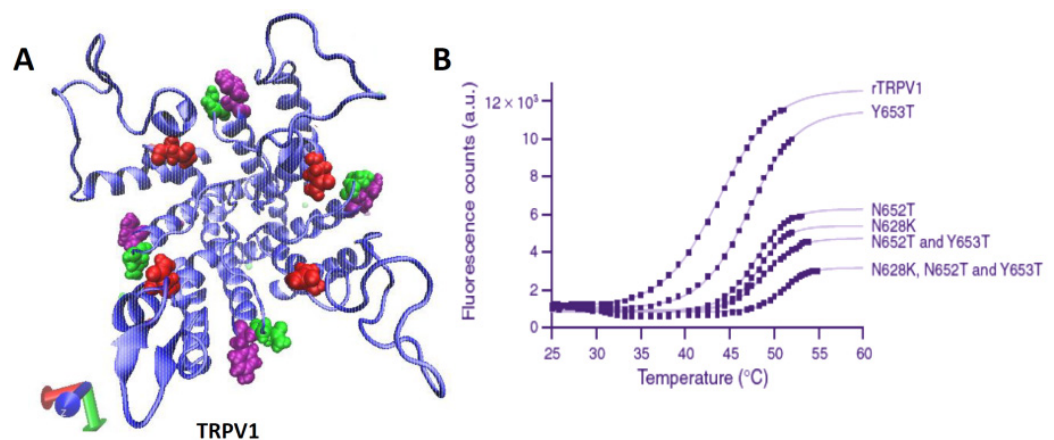

TRPV1
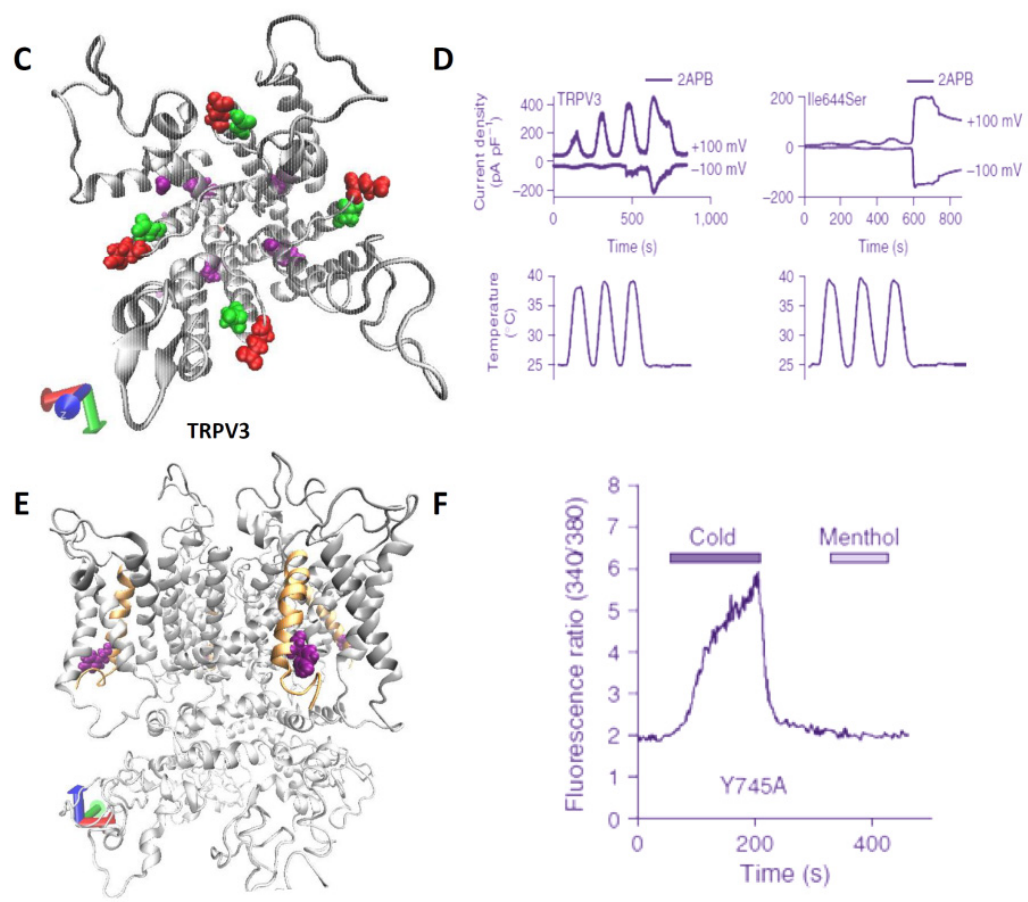

TRPM8

Figure 3. The high throughput screening technique used to identify key residues in thermoTRP channels. A. A top view of TRPV1 structure highlighting residues N628 (red), N652 (green), and Y653 (violet). B. A graph showing the normalized fluorescence change in response to an increase in temperature. The different mutants have a lower response compared with the wild type channel and the decrease in response is proportional to the number of residues mutated. C. Top view of the TRPV3 structure highlighting the residues I644 (red), Y661 (violet), and N647 (green). D. Responses to temperature changes of the wild type TRPV3 (left) and the I644S TRPV3 mutant. Notice that despite the fact that the temperature response in the mutant has been obliterated the response to the agonist $2 \mathrm{APB}$ is vey similar to that of the wild type channel. E. Lateral view of the TRPM8 structure showing the position of the residue Y745. F. The experiment shows that although the mutant is still sensitive to cold, it has lost its ability to respond to menthol. 
Finally, for TRPM8 the screening random mutagenesis technique was used to search for the residues constituting the menthol binding site [33]. Over a library of 14000 clones, Bandell et al. identified two mutations in TM2, $\mathrm{Y} 745 \mathrm{H}$ and L1009R, able to produce a failure in the menthol activation but keeping the other TRPM8 channel properties like voltagedependence, temperature sensitivity and $\mathrm{PIP}_{2}$ sensitivity intact. In the case of L1009R mutation, mutations by any other amino acid besides $R$ do not have any effect in the menthol activation showing us the power of this unbiased assay, very helpful when you don't have a crystal structure or an adequate molecular model of the channel.

\section{Physiological role of thermoTRP channels}

ThermoTRPs channels are key elements in many physiological processes. Powerful mutagenic tools such as knockout mice generation have been intensely used to study the physiological function of thermoTRP channels in vivo. In this section, we will summarize the information about the physiological role of thermoTRP channels, obtained from the study of the phenotype of knockout mice.

\subsection{TRPA1}

Transient Receptor Potential Ankiryn 1 (TRPA1) channel is activated by several pungent agents found in mustard oil, cinnamon and garlic, among others [36, 37]. This $\mathrm{Ca}^{2+}-$ permeable nonselective cation channel, expressed in a large subpopulation of nociceptors, was described to be activated by cold temperatures, $<17^{\circ} \mathrm{C}$ [38]. Thus, it was postulated to be the thermoreceptor for noxious cold temperatures in primary sensory neurons. This channel is activated by a large list of irritant substances, and nowadays it is considered the main molecular sensor of noxious chemical stimuli in the somatosensory system [39-41, reviewed in 42]. Two knockout mice of TRPA1 were described in 2006 [23, 39]. These mice were generated by targeted recombination that deleted the exons coding the pore region. Although disruption of the TRPA1 gene abolishes the behavioral responses to chemical activators of the channel, it has no effect in the response or prevalence of cold-sensitive primary neurons $[23,39]$. However, there are deficits in the response to mechanical stimuli $[23,43]$. Later it was found that like TRPV1 (see below), TRPA1 is actually involved in inflammatory-related hyperalgesia [44, 45]. Most likely, this role is given by its activation or modulation by a great variety of chemical agents, including several inflammatory- or cell damage-related molecules. Although TRPA1 seems to have a minor role in peripheral cold thermotransduction, a study with the knockout mice shows that this channel participates in the cold sensation in visceral nerves [46]. In vagal sensory neurons from the nodose ganglion, $\mathrm{TRPA}^{--}$mice have significantly less cold-sensitive neurons than wild type animals. Also the pharmacological profile of cold-evoked responses in neurons from nodose ganglia is compatible with a major role of TRPA1 in their cold sensitivity [46]. However, a more recent and detailed study of the phenotype of TRPA1 ${ }^{-/}$animals by Karashima and coworkers strongly suggest an important role of TRPA1 channels in noxious cold thermotransduction [47]. 


\subsection{TRPC5}

Transient Receptor Potential Canonical 5 (TRPC5) is a $\mathrm{Ca}^{2+}$-permeable nonselective cation channel expressed in the brain and several other tissues, including vascular smooth muscle cells, endothelial cells, adrenal medulla, mammary glands, yolk sac, activated T cells and monocytes and cardiac ventricles in hypertension (reviewed by [48]). Recently, it has been reported that TRPC5 displays a high sensitivity to cooling into the mild cold range [1]. This cold-dependent activation can be potentiated by activation of $\mathrm{G}_{\mathrm{q}}$-linked muscarinic type 1 receptor via carbachol, as well as by PLC activation via extracellular lanthanum [1]. The activation of TRPC5 by low temperatures, together with the fact that this channel is expressed in approximately $30 \%$ of mouse primary sensory neurons in culture, makes it a potential candidate to participate in cold sensing.

A TRPC5 deficient mouse was generated in 2009 [49]. However, from the data obtained using this TRPC5 deficient mice, it was difficult to assign a relevant role of TRPC5 in cold sensing. First, behavioural tests showed no differences between wild type and TRPC $5^{--}$mice in various temperature-sensing assays [1]. In contrast, in cultured primary sensory neurons, TRPC5 ${ }^{-/}$mice displayed a significant reduction in the percentage of cold-sensitive neurons and also an interesting reduction in TRPM8 channels detected by immunohistochemistry, with no changes in the nociceptive markers CGRP, IB 4 , NF200, peripherin, or TRPV1 protein. TRPC5-mediated currents could not be measured in this preparation of primary sensory neurons from dorsal root ganglia in wild type and knockout mice. These results suggest that TRPC5 activity in response to cold could be used for other adaptive or regulatory processes, such as localized metabolic changes, local vascular changes, retraction of nerve endings, or initiation of transcriptional programs [1]. Other possible explanation would be that the deletion of TRPC5 results in compensatory replacement by functionally overlapping of other cold transducers. This compensatory replacement, as it has been described for instance in tetrodotoxin-sensitive ion channels in Nav1.8-deficient mice [43], could result in the absence of a clear TRPC5 $\%$ phenotype in behavioural tests, and could explain the avoidance of cold temperatures displayed by TRPC5 ${ }^{--}$mice [1]. Further studies are needed to establish the possible role of TRPC5 in cold transduction and the physiological significance of the potentiation of its activity by cold temperatures.

\subsection{TRPM2}

Transient Receptor Potential Melastatin 2 (TRPM2), is an ion channel permeable to all physiological cations including $\mathrm{Ca}^{2+}$, which activation leads to an increase in intracellular $\mathrm{Ca}^{2+}$ concentration and/or membrane depolarization (reviewed by [48]). TRPM2 exhibits a widespread distribution that includes brain, bone marrow, spleen, heart, liver and lung, and also different cell types including immune cells (neutrophils, megakaryocytes, monocytes/macrophages), pancreatic $\beta$-cells and, endothelial cells, cardiomyocytes, microglia and neurons [48]. TRPM2 is activated by warm temperatures, with a threshold of $\sim 35^{\circ} \mathrm{C}$ [50]. This channel is not present in primary sensory neurons and a role in thermosensation has not been reported. 
TRPM2 contains a Nudix hydrolase (NudT9-H) domain in its C-terminus, that activates the channel through its binding with the adenosine 5'-diphosphoribose (ADPR)[48]. It has been described that TRPM2 is also activated by $\mathrm{H}_{2} \mathrm{O}_{2}$ and plays an important physiological role in regulation of the oxidative stress [51]. In addition, this channel can function as an intracellular channel $[52,53]$.

Knockout mice for these channels were generated by targeted homologous recombination in ES cells, disrupting the third exon of the TRPM2 gene [54]. Analysis of these animals evidenced that they are key regulators of intracellular calcium levels and they participate in signaling cascades related to the function of the immune system [54, 55]. TRPM2 also participates in the control of insulin secretion in pancreatic $\beta$ cells [56], making TRPM2-mice to have an impaired glucose metabolism because of low insulin secretion.

\subsection{TRPM3}

Transient Receptor Potential Melastatin 3 (TRPM3) is a $\mathrm{Ca}^{2+}$-permeable nonselective cation channel expressed in a range of different tissues, including brain, kidney, endocrine pancreas, ovary, and sensory neurons $[57,58]$. It has been reported that this channel is activated by hypotonic cell swelling, D-erytrosphingosine, strong depolarization, removal of extracellular $\mathrm{Na}^{+}$, and pregnenolone sulphate (reviewed by [59]). TRPM3, like other TRPM channels closely related such as TRPM2, TRPM4, TRPM5 and TRPM8, is a thermosensitive channel activated by heat [60].

Little is yet known about the physiological role of TRPM3 in vivo. In addition, the TRPM3 gene encodes several isoforms presenting different biophysical properties [61]. So far, this channel has been involved in the modulation of the secretion of insulin and interleukin-6, promotion of vascular contraction, and thermotransduction [60,62,63]. The generation of the deficient TRPM3 mice by homologous recombination by Vriens and colleagues, has been an important step to elucidate the physiological relevance of this channel. TRPM3 ${ }^{-/}$mice exhibited no obvious deficits in fertility, gross anatomy, body weight, core body temperature, locomotion, or exploratory behaviour. No differences in resting blood glucose were found, suggesting that basal glucose homeostasis is not affected. Thus, TRPM3 ${ }^{--}$mice appear generally healthy, with no indications of major developmental or metabolic deficits [60].

The thermosensitivity of TRPM3, its expression in sensory neurons, and the painful effect of the systemic administration of the activator of TRPM3 pregnenolone sulphate, allow to hypothesize about the potential role of TRPM3 in thermotransduction and nociception [60]. The analysis of primary sensory neurons from wild-type and TRPM3 ${ }^{--}$animals, showed a reduction in the percentage of heat sensitive neurons, and allowed to establish the existence of four distinct populations of heat-sensitive neurons. The largest population of the heat sensitive neurons responded to both pregnenolone sulphate (PS) and capsaicin, suggesting the coexpression of TRPM3 and TRPV1. The second most abundant population of heat positive neurons responded to PS but not to capsaicin (TRPM3-positive), and a minor fraction responded to capsaicin but not to PS (TRPV1-positive). Finally, a small percentage $(2 \%)$ of heat-activated neurons was unresponsive to both PS and capsaicin, indicating the existence of a TRPM3- and TRPV1-independent heat-sensing mechanism. 
The number of heat-sensitive neurons was reduced in a 25\% in TRPM3 ${ }^{-/-}$mice. The subgroup of heat-sensitive neurons responding to PS but not to capsaicin disappeared, whereas an increase was observed in the number of neurons that responded to heat and capsaicin and in the number of heat-positive neurons independent of TRPM3 and TRPV1. In agreement with these observations, the study of the heat responses of sensory neurons from the TRPM3 knockout mice in the presence of a specific antagonist of TRPV1, showed that an important fraction of heat sensitive neurons remained, pointing out the existence of another molecular entity responsible for the heat transduction [60]. The reduction in the population of heatsensitive neurons in TRPM3 ${ }^{--}$is consistent with a strong deficit in the detection of noxious heat stimuli displayed by these animals, as evidenced by prolonged reaction latencies in the tail immersion tests and in hot plate assays, and a reduced avoidance of hot temperatures. In addition, these mice show a significant and specific deficit in the nocifensive responses to TRPM3-activating stimuli, and a strong deficit in the development of inflammation-induced heat hyperalgesia [60]. Taken together, these results establish that TRPM3 works as a chemoand thermosensor in the somatosensory system, involved in the detection of noxious stimuli.

\subsection{TRPM4}

Transient Receptor Potential Melastatin 4 (TRPM4) is an ion channel selective for monovalent cations and no permeable to $\mathrm{Ca}^{2+}$. It is inhibited by intracellular free ATP and activated by internal $\mathrm{Ca}^{2+}$. This activation is modulated by ATP, $\mathrm{Ca}^{2+}$-calmodulin, and PKC. $\mathrm{PIP}_{2}$ also regulates the activity of the channel, by modulating its calcium and voltage sensitivity (reviewed by [64]). It has been described that heat, in the $15-35^{\circ} \mathrm{C}$ range, modulates the voltage sensitivity of TRPM4, resulting in an increase in current [65]. Despite of TRPM4 being detected in a large numbers of tissues such as heart, pancreas, placenta, and prostate and at lower levels in the kidney, skeletal muscle, liver, intestines, thymus, and spleen [64], it has not been reported in sensory neurons and thus it is very unlikely to have a role in thermosensation.

Knockout mice for TRPM4 were first generated by a Cre-loxP strategy that excised exons 15 and 16 of the gene (containing the first membrane-spanning segment of the protein), replacing them by a PGK-neo ${ }^{r}$ cassette that was removed by Cre recombinase [66]. A second study used the same strategy to target a region of the gene spanning from exons 3 to 6 [67]. Studies using the TRPM4 deficient mice show that this channel, like TRPM2, plays a role in controlling intracellular calcium levels during mast cell activation and dendritic cell migration [66-68]. It has also been shown that this ion channel helps to limit catecholamine release from chromaffin cells, indicating that TRPM4 $4^{--}$mice have an increased sympathetic tone and hypertension [69].

\subsection{TRPM5}

Transient Receptor Potential Melastatin 5 (TRPM5) is a cationic $\mathrm{Ca}^{2+}$-activated channel with an important role in vertebrate taste transduction. This channel was cloned by Perez and coworkers in 2002 [70]. TRPM5 is strongly expressed in taste cells where it is co-expressed 
with several taste-signaling molecules [70]. The transduction of sweet, bitter and amino acid tastes depends on the activation of $G$ protein-coupled membrane receptors that involve the participation of a common intracellular pathway. These receptors signal through a heterotrimeric $G$ protein that activates phospholipase $C \beta 2$, whose activation increase inositol 1,4,5-triphosphate $\left(\mathrm{IP}_{3}\right)$ levels, inducing $\mathrm{Ca}^{2+}$-release from intracellular stores that finally activates TRPM5 in the basolateral plasma membrane of taste receptor cells.

The first knockout mouse of TRPM5 was developed by Zhang and colleagues in 2003 [71], where exons 15 to 19 were replaced by the PGK-neomycin resistance cassette. This region encodes for the first five transmembrane domains and the pore region of the channel. These knockout animals are undistinguishable from the wild type animals in terms of weight, viability, general behavior and morphology and number of taste cells, taste receptors, and other signaling molecules. Nevertheless sweet, amino acid and bitter taste detection was completely abolished by TRPM5 ablation, with no effects on sour or salty tastes.

Rong and colleagues also generated a TRPM5 knockout mouse [72]. In this case, TRPM5 gene has a deletion of 2.4-kb of the $5^{\prime}$-flanking region of the gene. This deletion includes the promoter and coding region encompassing exons 1 to 4 . Using this genetically modified mouse, Talavera and colleagues studied the temperature sensitivity of TRPM5, and they found that this is a heat activated channel [65]. Modulation by temperature of the human perception of different taste modalities is a well known fact, and temperature dependence of TRPM5 appears to contribute to this phenomenon in vivo. By using this animal model, Talavera and coworkers demonstrated that heat potentiates the gustatory (chorda tympani) nerve responses to sweet compounds in wild type animals but not in TRPM5 ${ }^{-/}$mice [65]. Molecular ablation of TRPM5 also eliminates, to a large extent, the responses to natural and artificial sweet compounds recorded in chorda tympani nerve. Interestingly, the responses to umami and bitter tastants were not potentiated by heat in both wild type and knockout animals. The thermal sensitivity of TRPM5 could explain the stronger perceived sweetness of sweet beverages at warmer temperatures in humans. On the other hand, the direct modulation of TRPM5 by temperature could explain why heating or cooling of the tongue can evoke sensations of taste.

\subsection{TRPM8}

The Transient Receptor Potential Melastatin 8 (TRPM8) channel is the main molecular entity responsible for the transduction of moderate cold in the somatosensory system (reviewed in $[73,74])$. This $\mathrm{Ca}^{2+}$-permeable cation channel, identified in 2001 as a protein up-regulated in prostate cancer [75] and cloned by two groups independently in 2002 [76, 77], is activated not only by cold but also by chemical cooling compounds such as menthol and by voltage $[3,6,76$, 77]. This channel is expressed mainly in dorsal root and trigeminal ganglia, but its expression has been detected in several other tissues, where its function is under intense study [73, 74].

Knockout mice lacking functional expression of TRPM8 were generated by three groups independently in 2007 [78-80]. Survival and general appearance of these mutant mice are normal, with no differences in the mean core body temperatures compared to wild type. 
However, the cold sensitivity of all these mutants is strongly compromised, especially in the range of innocuous cold temperatures. Bautista and coworkers [78] generated a TRPM8 knockout mice by deletion of the coding region between residues 594 and 661, into the large intracellular N-terminal domain of TRPM8, introducing a stop codon before and frameshift after this segment. The non-functional truncated transcript produced by TRPM8 ${ }^{-/}$mice allowed to confirm by in situ hybridization that the loss of TRPM8 functional protein does not eliminate those neurons that normally express the channel. Behavioral studies revealed that these mice present a profound impairment to discriminate between cold and warm environments. Electrophysiological recordings of single sensory fibers from TRPM8 knockout mice show not only a strongly reduced sensitivity to innocuous cold stimuli in the low-threshold cold sensitive fibers, but also a marked reduction in the responses to temperature reductions in the high-threshold cold sensitive afferent neurons. Cold sensitive C- fibers of these animals present a reduced basal firing rate, indicating that TRPM8 is also important for the generation of the basal action potential firing of these neurons at resting temperatures. The molecular ablation of TRPM8 does not affect the conduction velocity and electrical activation threshold of these fibers. Calcium imaging experiments in cultured sensory neurons from TRPM8 ${ }^{--}$mice show a decrease in both number and magnitude of the responses to cold, completing a picture where TRPM8 appears as the main molecular entity responsible for the cold sensitivity, with a role not only in the transduction of innocuous cold but also in the detection of cold in the noxious range.

Mice lacking functional expression of TRPM8 have been also generated by Colburn and coworkers by using homologous recombination [79]. These knockout mice are completely normal regarding general behaviors, lifespan and fertility. TRPM8 ${ }^{--}$mice show a complete absence of behavioral responses to systemic chemical activation of TRPM8 by icilin, the strongest synthetic activator described for this channel so far. On the other hand, in a test to evaluate thermal preference, TRPM8 ${ }^{-/}$mice spend the same fraction of time in cold surfaces than in comfortable warm ones, unlike wild type animals that prefer warm surfaces. The development of neuropathic cold-induced pain was also strongly reduced by the genetic ablation of the channel in these animals. TRPM8 gene disruption also reduces cold sensitivity of primary sensory neurons in culture, studied by calcium imaging [79].

Dhaka and coworkers have also developed a TRPM8 knockout mouse. They used a targeting construct to delete amino acids in the N-terminal region knocked in by using a farnesylated enhanced green fluorescent protein (EGFP-F) followed by an SV40polyA tail in frame with the start codon of the channel [80]. This SV40polyA tail prevents the transcription of TRPM8, and EGFP allows to identify neurons that would express the channel in normal conditions. These TRPM8-deficient mice are completely viable, and they exhibit a strongly reduced avoidance to cold in two-temperature assays and in thermotaxis assays of temperature gradients. Molecular ablation of TRPM8 also reduced the responses to cooling chemicals. Using this mouse and a preparation that allows to record the electrical activity of unitary cold-sensitive nerve endings in the cornea, Parra and colleagues demonstrated that not only the responses to cold but also the ongoing electrical activity of these sensory neurons was proportional to the functional expression level of TRPM8 channels [81]. Altogether, these evidences confirm a critical role of TRPM8 in cold thermosensitivity. 


\subsection{TRPV1}

The Transient Receptor Potential Vanilloid 1 (TRPV1), a $\mathrm{Ca}^{2+}$-permeable nonselective cation channel, was the first thermoTRP channel to be cloned and the first protein to be shown to have such an unusually high dependence on temperature [82]. Its open probability increases with heat with a threshold of around $43^{\circ} \mathrm{C}$ (in heterologous expression systems), as well as upon exposure to capsaicin (the active compound in chili peppers) or low $\mathrm{pH}$. Given its properties as well as its presence in a population of primary sensory neurons from the dorsal root and trigeminal ganglia, it was hypothesized as the main responsible for heat sensory transduction in mammals. In the year 2000, two groups were able to generate knock-out mice for the TRPV1 gene by targeted homologous recombination [83, 84]. Both works reported absence of heat-, capsaicin- or acid-evoked currents in cultured sensory neurons from TRPV1 ${ }^{-/}$mice. Moreover Caterina et al. [83] reported an impaired physiological and behavioral response to capsaicin in the knockout animals: while a normal animal would avoid consumption of a capsaicin-containing solution and decrease its body temperature upon capsaicin administration, mice with a disrupted TRPV1 gene lacked these responses. However the most expected prediction was not fulfilled in behavioral tests, as TRPV1 ${ }^{--}$animals can detect non-noxious heat as wild type animals do. Instead, a deficit was detected in the knock-out animals regarding their response to noxious heat $\left(>50^{\circ} \mathrm{C}\right)$ stimuli, a result that was striking given the threshold of $43^{\circ} \mathrm{C}$ for channel activation. This result can be explained assuming that the TRPV2 channel (see below) takes the place of TRPV1, however, the heat-responding neurons in TRPV1 1 mice do not express TRPV2 [85].

In addition, what was actually found in TRPV1/- mice was a deficit in heat hyperalgesia [83, 84]. This process consists in the sensitization of the pain receptors upon persistence of the pain stimulus. The noxious heat in this case, may involve different inflammatory signaling cascades that lead to a sensitization of TRPV1 either by decreasing PIP2 levels, activation of PKC, activation of PKA, and/or an increase of the number of TRPV1 channels in the plasma membrane [86].

Regarding body temperature $\left(\mathrm{T}_{\mathrm{B}}\right)$ control, mice lacking this channel have a wider daily $\mathrm{T}_{\mathrm{B}}$ cycle compared to wild type animals, with lower Тв during the day (inactivity period), and higher Тв during the night (active phase) [87, 88]. However, they still display a normal heat tolerance, i.e., their body temperature increases as much as in normal mice when exposed to a hot ambient [87]. On the contrary, when the TRPV1 channels of wild type mice are desensitized with capsaicin, these animals show a robust heat-intolerance [87], evidencing that knockout mice give only a partial picture of the role of TRPV1 as a heat sensor in physiological conditions.

\subsection{TRPV2}

The Transient Receptor Potential Vanilloid 2 (TRPV2) is activated by higher temperatures than TRPV1, with a threshold of $\sim 52^{\circ} \mathrm{C}$ [89], and by cannabinoids[30]. The role of this 
channel is still poorly understood and although it is expressed in sensory ganglia no sensory function has yet been established. Instead, it has been associated to the axon outgrowth of developing DRG and motor neurons [90]. Knockout mice were generated by a loxP recombination that disrupted 4 exons comprising the $5^{\text {th }} \mathrm{TM}$ segment, the pore loop and the $6^{\text {th }}$ TM segment [91]. These mice have unimpaired responses to heat and mechanical stimuli; however they were reported to be susceptible to perinatal lethality and to have a reduced body weight [91]. When looking at macrophage function [92], it was found that TRPV2 has a critical role in the early stages of phagocytosis. The channel is recruited to nascent phagosomes where it depolarizes the membrane initiating a signal cascade that results in clustering of phagocytic receptors. Moreover, macrophages from TRPV2 ${ }^{-/}$mice have a diminished motility [92].

\subsection{TRPV3}

The Transient Receptor Potential Vanilloid 3 (TRPV3) is a $\mathrm{Ca}^{2+}$-permeable cation channel activated by innocuous warm temperatures $\left(\geq 33^{\circ} \mathrm{C}\right)$ [93]. This channel is highly expressed in skin keratinocytes and oral and nasal epithelia, while the expression levels of TRPV3 in brain, sensory ganglia and spinal cord are in general weak [94]. TRPV3 can be activated by a large list of chemicals such as eugenol, thymol, and carvacrol, savory, clove, thyme, camphor, and 2-aminoethoxydiphenyl borate [94, 95]. Heat activation of TRPV3 shows a marked sensitization under repeated heat stimulation, both in recombinant systems and in keratinocytes.

The first knockout mouse for TRPV3 was developed by Moqrich and coworkers [95]. Cultured keratinocytes from these wild type mice show sensitization to repeated heat stimulation, responses and sensitization to camphor stimuli, and blockage by ruthenium red. Keratinocytes from TRPV3 ${ }^{-/}$mice did not respond to camphor or heat stimuli [95]. The most outstanding results that pointed out TRPV3 as a molecular sensor of the thermal stimuli in the warm range were obtained from behavioral experiments in TRPV3 ${ }^{--}$mice. TRPV3-deficient mice showed a reduced tendency to migrate towards warm surfaces and a defect in their responses to noxious heat stimulation [95]. The remaining sensitivity to warm temperatures in TRPV3 ${ }^{--}$mice implies that other molecular entities are also involved in innocuous warm thermosensation. On the other hand, if skin keratinocytes participate in the detection of warm temperatures at the surface of the skin, a mechanism to transmit the information from keratinocytes to sensory nerves is needed. In this line, it has been proposed that ATP released from keratinocytes due the activation of TRPV3 channels under warm temperature stimulation, could potentially signal to a variety of P2X- or P2Yexpressing sensory nerve terminals within the epidermis to transmit thermal information [96]. In addition to their role in thermosensation, several rodent strains bearing mutant TRPV3, such as the autosomal dominant DS-Nh (no-hair) mouse and the WBN/Kob-Ht rat, are spontaneously hairless and develop atopic dermatitis-like lesions. These two strains present a point mutation in the S4-S5 linker of TRPV3 (G573S in DS-Nh and G573C in WBN/Kob-Ht mice) [97]. The study of these point mutations, using a recombinant system, showed that a single substitution of the glycine 573 results in a constitutive active channel, 
insensitive to thermal and chemical stimuli [98]. In addition to these hairless strains, the TRPV3 deficient mice also display a hair abnormality. In the first description of TRPV3 deficient mice, a subtle and temporary hair irregularity in the abdominal area was reported around the third postnatal week [95]. However, in a more exhaustive study about the relationship between TRPV3 and hair morphogenesis, using another TRPV3-- strain [99], the authors found important phenotypic changes related to abnormalities in skin, hair, and whiskers. The results of this work show that TRPV3-deficient mice displayed a deregulation of TGF- $\alpha$ /EGFR signaling that affect keratinocyte terminal differentiation, affecting hair generation [99].

\subsection{TRPV4}

Transient Receptor Potential Vanilloid 4 (TRPV4) is a $\mathrm{Ca}^{2+}$-permeable cation channel that was cloned in 2000 by two groups independently [100, 101]. Originally identified as an osmosensitive channel, TRPV4 is expressed in kidney, lung, spleen, testis, tongue, fat cells, keratinocytes, inner and outer hair cells of the organ of Corti, sensory ganglia and central nervous system [100, 102-104]. In heterologous expression systems, TRPV4 show a threshold temperature of activation near to $34^{\circ} \mathrm{C}$, a temperature close to the resting temperature of the skin [103], suggesting that TRPV4 could work as a warm detector in physiological conditions. A TRPV4 knockout mouse was developed and described by Suzuki and colleagues in 2003 [104]. TRPV4 gene disruption was achieved by homologous recombination, using a neomycin resistance (PKG-neo) cassette to replace the region encoding exon 4. Behavioral studies show that mice lacking TRPV4 did not display any impairment to sense noxious heat [104], but deficiencies in thermal selection between wild type and knockout mice were evident in temperature selection tests in the range of warm comfortable temperatures [105]. Interestingly, electrophysiological recordings of sensory neurons in the femoral nerve show a decrease in the warmth-dependent electrical activity in TRPV4 ${ }^{-/}$mice. Moreover, molecular ablation of TRPV4 also increased the latency to escape from hot (from 35 to $50^{\circ} \mathrm{C}$ ) in animals with carrageenan-induced heat hypersensitivity [106], suggesting an important role for TRPV4 in both detection of warm temperatures and thermal hyperalgesia.

In Table 1 we summarize the information obtained so far from the study of the phenotype of knockout mice of thermoTRP channels.

\section{Conclusions}

Mutagenesis combined with electrophysiological studies have been fundamental in the understanding of the molecular mechanisms involved in the gating of temperatureactivated TRP channels. The evidence accumulated from the molecular cloning of TRPV1 channel until nowadays, points out to the thermoTRP channels not only as predominant sensors of thermal and noxious stimuli in the somatosensory system in vivo, but also as key players in many other physiological processes. 
ThermoTRP Temperature Knockout mice phenotype and physiological role channel sensitivity

\begin{tabular}{|c|c|c|}
\hline TRPA1 & $\leq 17{ }^{\circ} \mathrm{C}$ & $\begin{array}{l}\text { Loss of sensitivity to pungent natural compounds, } \\
\text { environmental irritants and proalgesic agents. No effects in } \\
\text { cold sensation of peripheral receptors. Deficits in cold } \\
\text { sensation at vagal sensory neurons. Reduced hyperalgesia to } \\
\text { mechanical stimuli. } \\
\text { Mediator of inflammatory-related hyperalgesia. Contribution } \\
\text { to noxious cold transduction. Cold transductor in visceral } \\
\text { nerves. }\end{array}$ \\
\hline TRPM8 & $\leq 34^{\circ} \mathrm{C}$ & $\begin{array}{l}\text { Impaired detection of innocuous and noxious cold. } \\
\text { Suppression of the ongoing activity of cold thermoreceptor } \\
\text { fibers. Strong reduction of cold-sensitive neurons in culture. } \\
\text { Main molecular sensor to cold in the somatosensory system. }\end{array}$ \\
\hline TRPC5 & $\leq 37^{\circ} \mathrm{C}$ & $\begin{array}{l}\text { No temperature-sensitive behavioural changes. Gain of } \\
\text { function in C-cold nociceptors. Reduction in TRPM8 } \\
\text { expressing cells and cold-sensitive neurons. } \\
\text { Role in the detection and local adaptation to cold temperatures } \\
\text { in the peripheral nervous system. }\end{array}$ \\
\hline
\end{tabular}

TRPM2 $\quad 35-45^{\circ} \mathrm{C} \quad$ Impaired immune response. Impaired insulin secretion and glucose metabolism.

$\mathrm{Ca}^{2+}$ influx through TRPM2 controls signaling cascades responsible for chemokine production.

Involved in insulin secretion stimulated by glucose.

TRPM4 $\quad 15-35{ }^{\circ} \mathrm{C} \quad$ Increased IgE-dependent mast cell activation, impaired chemokine-dependent migration of dendritic cells. Impaired catecholamine release from chromaffine cells leading to increased sympathetic tone and hypertension.

Involved in intracellular calcium regulation.

TRPM5 $\quad 15-35^{\circ} \mathrm{C} \quad$ Impaired detection of sweet, bitter and umami tastants. Key molecular component of taste transduction machinery. Proposed molecular counterpart of the modulation by temperature of the human perception of different taste modalities.

TRPM3 $\quad 25-40{ }^{\circ} \mathrm{C} \quad$ Impaired detection of noxious heat stimuli. Deficit in the development of heat hyperalgesia. Reduction in the percentage of heat positive neurons in dorsal root and trigeminal ganglia. Chemo- and thermosensor in the somatosensory system, involved in the detection of noxious stimuli in healthy and inflamed tissue. 


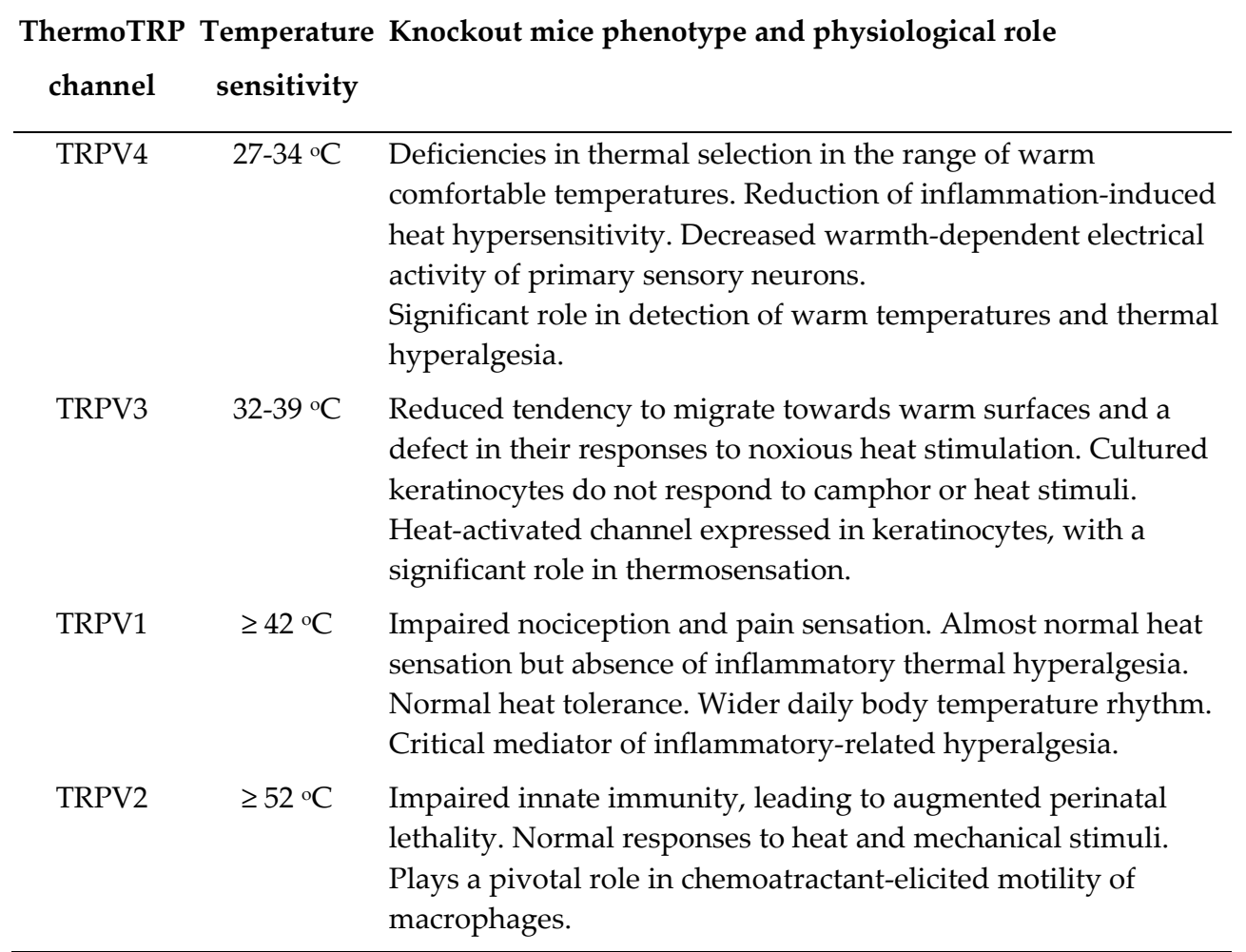

Table 1. The thermoTRP channels. Table summarizing the temperature sensitivity, knockout mice phenotype, and physiological role of mammalian thermoTRP channels.

\section{Author details}

María Pertusa and Rodolfo Madrid

Laboratorio de Neurociencia, Departamento de Biología, Facultad de Química y Biología, Universidad de Santiago de Chile, Santiago, Chile

Ramón Latorre ${ }^{*}$ and Patricio Orio

Centro Interdisciplinario de Neurociencia de Valparaíso, Facultad de Ciencias, Universidad de Valparaíso, Valparaíso, Chile

Hans Moldenhauer

Centro Interdisciplinario de Neurociencia de Valparaíso, Facultad de Ciencias, Universidad de Valparaíso, Valparaíso, Chile

Programa de Doctorado en Ciencias mención Neurociencias, Facultad de Ciencias, Universidad de Valparaíso, Valparaíso, Chile

${ }^{*}$ Corresponding Author 
Sebastián Brauchi

Instituto de Fisiología, Universidad Austral de Chile, Valdivia, Chile

\section{Acknowledgement}

Authors are supported by Fondecyt Grants 1110430 to R. Latorre; 1110906 to S. Brauchi; 1100983 to R. Madrid; 11090308 to P. Orio; 3110128 to M. Pertusa. R. Madrid thanks the support of Vicerrectoría de Investigación y Desarrollo of the Universidad of Santiago de Chile. H. Moldenhauer is recipient of a Conicyt Ph.D. Fellowship. The Centro Interdisciplinario de Neurociencia de Valparaíso is supported by the Millenium Science Initiative of the Ministry of Economy (Chile).

\section{References}

[1] Zimmermann, K., et al., Transient receptor potential cation channel, subfamily $C$, member 5 (TRPC5) is a cold-transducer in the peripheral nervous system. Proceedings of the National Academy of Sciences of the United States of America, 2011. 108(44): p. 18114-9.

[2] Basbaum, A.I., et al., Cellular and molecular mechanisms of pain. Cell, 2009. 139(2): p. 26784.

[3] Brauchi, S., P. Orio, and R. Latorre, Clues to understanding cold sensation: thermodynamics and electrophysiological analysis of the cold receptor TRPM8. Proc Natl Acad Sci U S A, 2004. 101(43): p. 15494-9.

[4] Latorre, R., et al., ThermoTRP channels as modular proteins with allosteric gating. Cell calcium, 2007. 42(4-5): p. 427-38.

[5] Liu, B., K. Hui, and F. Qin, Thermodynamics of heat activation of single capsaicin ion channels VR1. Biophysical journal, 2003. 85(5): p. 2988-3006.

[6] Voets, T., et al., The principle of temperature-dependent gating in cold-and heat-sensitive TRP channels. Nature, 2004. 430(7001): p. 748-54.

[7] Yao, J., B. Liu, and F. Qin, Kinetic and energetic analysis of thermally activated TRPV1 channels. Biophysical journal, 2010. 99(6): p. 1743-53.

[8] Zakharian, E., C. Cao, and T. Rohacs, Gating of transient receptor potential melastatin 8 (TRPM8) channels activated by cold and chemical agonists in planar lipid bilayers. J Neurosci, 2010. 30(37): p. 12526-34.

[9] Tanford, C., Protein denaturation. Advances in protein chemistry, 1968. 23: p. 121-282.

[10] Cordero-Morales, J.F., E.O. Gracheva, and D. Julius, Cytoplasmic ankyrin repeats of transient receptor potential A1 (TRPA1) dictate sensitivity to thermal and chemical stimuli. Proceedings of the National Academy of Sciences of the United States of America, 2011. 108(46): p. E1184-91.

[11] Yao, J., B. Liu, and F. Qin, Modular thermal sensors in temperature-gated transient receptor potential (TRP) channels. Proceedings of the National Academy of Sciences of the United States of America, 2011. 108(27): p. 11109-14.

[12] Grandl, J., et al., Pore region of TRPV3 ion channel is specifically required for heat activation. Nature neuroscience, 2008. 11(9): p. 1007-13. 
[13] Grandl, J., et al., Temperature-induced opening of TRPV1 ion channel is stabilized by the pore domain. Nature neuroscience, 2010. 13(6): p. 708-14.

[14] Brauchi, S., et al., Dissection of the components for PIP2 activation and thermosensation in TRP channels. Proceedings of the National Academy of Sciences of the United States of America, 2007. 104(24): p. 10246-51.

[15] Vlachova, V., et al., Functional role of C-terminal cytoplasmic tail of rat vanilloid receptor 1. The Journal of neuroscience : the official journal of the Society for Neuroscience, 2003. 23(4): p. 1340-50.

[16] Brauchi, S., et al., A hot-sensing cold receptor: C-terminal domain determines thermosensation in transient receptor potential channels. J Neurosci, 2006. 26(18): p. 4835-40.

[17] Schreiber, M., A. Yuan, and L. Salkoff, Transplantable sites confer calcium sensitivity to BK channels. Nature neuroscience, 1999. 2(5): p. 416-21.

[18] Jiang, Y., et al., Structure of the RCK domain from the E. coli K+ channel and demonstration of its presence in the human BK channel. Neuron, 2001. 29(3): p. 593-601.

[19] Choe, S., Potassium channel structures. Nature reviews. Neuroscience, 2002. 3(2): p. 11521.

[20] Zagotta, W.N., et al., Structural basis for modulation and agonist specificity of HCN pacemaker channels. Nature, 2003. 425(6954): p. 200-5.

[21] Long, S.B., E.B. Campbell, and R. Mackinnon, Voltage sensor of Kv1.2: structural basis of electromechanical coupling. Science, 2005. 309(5736): p. 903-8.

[22] Murata, Y., et al., Phosphoinositide phosphatase activity coupled to an intrinsic voltage sensor. Nature, 2005. 435(7046): p. 1239-43.

[23] Nieto-Posadas, A., A. Jara-Oseguera, and T. Rosenbaum, TRP channel gating physiology. Curr Top Med Chem, 2011. 11(17): p. 2131-50.

[24] Rohacs, T., Phosphoinositide regulation of non-canonical transient receptor potential channels. Cell Calcium, 2009. 45(6): p. 554-65.

[25] Yang, F., et al., Thermosensitive TRP channel pore turret is part of the temperature activation pathway. Proceedings of the National Academy of Sciences of the United States of America, 2010. 107(15): p. 7083-8.

[26] Yang, F., et al., Reply to Yao et al.: Is the pore turret just thermoTRP channels' appendix? Proceedings of the National Academy of Sciences, 2010. 107(32): p. E126-E127.

[27] Yao, J., B. Liu, and F. Qin, Pore turret of thermal TRP channels is not essential for temperature sensing. Proceedings of the National Academy of Sciences of the United States of America, 2010. 107(32): p. E125; author reply E126-7.

[28] Latorre, R., C. Zaelzer, and S. Brauchi, Structure-functional intimacies of transient receptor potential channels. Q Rev Biophys, 2009. 42(3): p. 201-46.

[29] Lu, G., et al., TRPV1b, a functional human vanilloid receptor splice variant. Molecular pharmacology, 2005. 67(4): p. 1119-27.

[30] Neeper, M.P., et al., Activation properties of heterologously expressed mammalian TRPV2: evidence for species dependence. The Journal of biological chemistry, 2007. 282(21): p. 15894-902. 
[31] Clapham, D.E. and C. Miller, A thermodynamic framework for understanding temperature sensing by transient receptor potential (TRP) channels. Proceedings of the National Academy of Sciences of the United States of America, 2011. 108(49): p. 19492-7.

[32] Sullivan, E., E.M. Tucker, and I.L. Dale, Measurement of [Ca2+] using the Fluorometric Imaging Plate Reader (FLIPR). Methods in molecular biology, 1999. 114: p. 125-33.

[33] Bandell, M., et al., High-throughput random mutagenesis screen reveals TRPM8 residues specifically required for activation by menthol. Nat Neurosci, 2006. 9(4): p. 493-500.

[34] Chung, M.K., et al., 2-aminoethoxydiphenyl borate activates and sensitizes the heat-gated ion channel TRPV3. The Journal of neuroscience : the official journal of the Society for Neuroscience, 2004. 24(22): p. 5177-82.

[35] Hu, H.Z., et al., 2-aminoethoxydiphenyl borate is a common activator of TRPV1, TRPV2, and TRPV3. The Journal of biological chemistry, 2004. 279(34): p. 35741-8.

[36] Bandell, M., et al., Noxious cold ion channel TRPA1 is activated by pungent compounds and bradykinin. Neuron, 2004. 41(6): p. 849-57.

[37] Bautista, D.M., et al., Pungent products from garlic activate the sensory ion channel TRPA1. Proceedings of the National Academy of Sciences of the United States of America, 2005. 102(34): p. 12248-52.

[38] Story, G.M., et al., ANKTM1, a TRP-like channel expressed in nociceptive neurons, is activated by cold temperatures. Cell, 2003. 112(6): p. 819-29.

[39] Bautista, D.M., et al., TRPA1 mediates the inflammatory actions of environmental irritants and proalgesic agents. Cell, 2006. 124(6): p. 1269-82.

[40] Macpherson, L.J., et al., An ion channel essential for sensing chemical damage. The Journal of neuroscience : the official journal of the Society for Neuroscience, 2007. 27(42): p. 11412-5.

[41] Andersson, D.A., et al., Transient receptor potential A1 is a sensory receptor for multiple products of oxidative stress. The Journal of neuroscience : the official journal of the Society for Neuroscience, 2008. 28(10): p. 2485-94.

[42] Venkatachalam, K. and C. Montell, TRP channels. Annu Rev Biochem, 2007. 76: p. 387-417.

[43] Zimmermann, K., et al., Sensory neuron sodium channel Nav1.8 is essential for pain at low temperatures. Nature, 2007. 447(7146): p. 855-8.

[44] Petrus, M., et al., A role of TRPA1 in mechanical hyperalgesia is revealed by pharmacological inhibition. Molecular pain, 2007. 3: p. 40.

[45] Fernandes, E.S., et al., A distinct role for transient receptor potential ankyrin 1, in addition to transient receptor potential vanilloid 1, in tumor necrosis factor alpha-induced inflammatory hyperalgesia and Freund's complete adjuvant-induced monarthritis. Arthritis and rheumatism, 2011. 63(3): p. 819-29.

[46] Fajardo, O., et al., TRPA1 channels mediate cold temperature sensing in mammalian vagal sensory neurons: pharmacological and genetic evidence. The Journal of neuroscience : the official journal of the Society for Neuroscience, 2008. 28(31): p. 7863-75.

[47] Karashima, Y., et al., TRPA1 acts as a cold sensor in vitro and in vivo. Proceedings of the National Academy of Sciences of the United States of America, 2009. 106(4): p. 1273-8.

[48] Jiang, L.H., N. Gamper, and D.J. Beech, Properties and therapeutic potential of transient receptor potential channels with putative roles in adversity: focus on TRPC5, TRPM2 and TRPA1. Current drug targets, 2011. 12(5): p. 724-36. 
[49] Riccio, A., et al., Essential role for TRPC5 in amygdala function and fear-related behavior. Cell, 2009. 137(4): p. 761-72.

[50] Togashi, K., et al., TRPM2 activation by cyclic ADP-ribose at body temperature is involved in insulin secretion. The EMBO journal, 2006. 25(9): p. 1804-15.

[51] Takahashi, N., et al., Roles of TRPM2 in oxidative stress. Cell Calcium, 2011. 50(3): p. 27987.

[52] Patel, S. and R. Docampo, In with the TRP channels: intracellular functions for TRPM1 and TRPM2. Science signaling, 2009. 2(95): p. pe69.

[53] Sumoza-Toledo, A. and R. Penner, TRPM2: a multifunctional ion channel for calcium signalling. The Journal of physiology, 2011. 589(Pt 7): p. 1515-25.

[54] Yamamoto, S., et al., TRPM2-mediated Ca2+influx induces chemokine production in monocytes that aggravates inflammatory neutrophil infiltration. Nature medicine, 2008. 14(7): p. 738-47.

[55] Knowles, H., et al., Transient Receptor Potential Melastatin 2 (TRPM2) ion channel is required for innate immunity against Listeria monocytogenes. Proceedings of the National Academy of Sciences of the United States of America, 2011. 108(28): p. 11578-83.

[56] Uchida, K., et al., Lack of TRPM2 impaired insulin secretion and glucose metabolisms in mice. Diabetes, 2011. 60(1): p. 119-26.

[57] Harteneck, C. and G. Schultz, TRPV4 and TRPM3 as Volume-Regulated Cation Channels, in TRP Ion Channel Function in Sensory Transduction and Cellular Signaling Cascades, W.B. Liedtke and S. Heller, Editors. 2007: Boca Raton (FL).

[58] Staaf, S., et al., Dynamic expression of the TRPM subgroup of ion channels in developing mouse sensory neurons. Gene expression patterns : GEP, 2010. 10(1): p. 65-74.

[59] Nilius, B. and T. Voets, A TRP channel-steroid marriage. Nature cell biology, 2008. 10(12): p. 1383-4.

[60] Vriens, J., et al., TRPM3 is a nociceptor channel involved in the detection of noxious heat. Neuron, 2011. 70(3): p. 482-94.

[61] Oberwinkler, J., et al., Alternative splicing switches the divalent cation selectivity of TRPM3 channels. The Journal of biological chemistry, 2005. 280(23): p. 22540-8.

[62] Naylor, J., et al., Pregnenolone sulphate- and cholesterol-regulated TRPM3 channels coupled to vascular smooth muscle secretion and contraction. Circulation research, 2010. 106(9): p. 1507-15.

[63] Wagner, T.F., et al., Transient receptor potential M3 channels are ionotropic steroid receptors in pancreatic beta cells. Nature cell biology, 2008. 10(12): p. 1421-30.

[64] Guinamard, R., M. Demion, and P. Launay, Physiological roles of the TRPM4 channel extracted from background currents. Physiology, 2010. 25(3): p. 155-64.

[65] Talavera, K., et al., Heat activation of TRPM5 underlies thermal sensitivity of sweet taste. Nature, 2005. 438(7070): p. 1022-5.

[66] Vennekens, R., et al., Increased IgE-dependent mast cell activation and anaphylactic responses in mice lacking the calcium-activated nonselective cation channel TRPM4. Nature immunology, 2007. 8(3): p. 312-20. 
[67] Barbet, G., et al., The calcium-activated nonselective cation channel TRPM4 is essential for the migration but not the maturation of dendritic cells. Nature immunology, 2008. 9(10): p. 1148-56.

[68] Shimizu, T., et al., TRPM4 regulates migration of mast cells in mice. Cell Calcium, 2009. 45(3): p. 226-32.

[69] Mathar, I., et al., Increased catecholamine secretion contributes to hypertension in TRPM4deficient mice. The Journal of clinical investigation, 2010. 120(9): p. 3267-79.

[70] Perez, C.A., et al., A transient receptor potential channel expressed in taste receptor cells. Nature neuroscience, 2002. 5(11): p. 1169-76.

[71] Zhang, Y., et al., Coding of sweet, bitter, and umami tastes: different receptor cells sharing similar signaling pathways. Cell, 2003. 112(3): p. 293-301.

[72] Damak, S., et al., Trpm5 null mice respond to bitter, sweet, and umami compounds. Chemical senses, 2006. 31(3): p. 253-64.

[73] Babes, A., et al., TRPM8, a Sensor for Mild Cooling in Mammalian Sensory Nerve Endings. Curr Pharm Biotechnol, 2011. 12(1): p. 78-88.

[74] Latorre, R., et al., A cool channel in cold transduction. Physiology, 2011. 26(4): p. 273-85.

[75] Tsavaler, L., et al., Trp-p8, a novel prostate-specific gene, is up-regulated in prostate cancer and other malignancies and shares high homology with transient receptor potential calcium channel proteins. Cancer Res, 2001. 61(9): p. 3760-9.

[76] McKemy, D.D., W.M. Neuhausser, and D. Julius, Identification of a cold receptor reveals a general role for TRP channels in thermosensation. Nature, 2002. 416(6876): p. 52-8.

[77] Peier, A.M., et al., A TRP channel that senses cold stimuli and menthol. Cell, 2002. 108(5): p. 705-15.

[78] Bautista, D.M., et al., The menthol receptor TRPM8 is the principal detector of environmental cold. Nature, 2007. 448(7150): p. 204-8.

[79] Colburn, R.W., et al., Attenuated cold sensitivity in TRPM8 null mice. Neuron, 2007. 54(3): p. 379-86.

[80] Dhaka, A., et al., TRPM8 is required for cold sensation in mice. Neuron, 2007. 54(3): p. 371-8.

[81] Parra, A., et al., Ocular surface wetness is regulated by TRPM8-dependent cold thermoreceptors of the cornea. Nat Med, 2010. 16(12): p. 1396-9.

[82] Caterina, M.J., et al., The capsaicin receptor: a heat-activated ion channel in the pain pathway. Nature, 1997. 389(6653): p. 816-24.

[83] Caterina, M.J., et al., Impaired nociception and pain sensation in mice lacking the capsaicin receptor. Science, 2000. 288(5464): p. 306-13.

[84] Davis, J.B., et al., Vanilloid receptor-1 is essential for inflammatory thermal hyperalgesia. Nature, 2000. 405(6783): p. 183-7.

[85] Woodbury, C.J., et al., Nociceptors lacking TRPV1 and TRPV2 have normal heat responses. The Journal of neuroscience : the official journal of the Society for Neuroscience, 2004. 24(28): p. 6410-5.

[86] Zhang, X. and P.A. McNaughton, Why pain gets worse: the mechanism of heat hyperalgesia. The Journal of general physiology, 2006. 128(5): p. 491-3.

[87] Szelenyi, Z., et al., Daily body temperature rhythm and heat tolerance in TRPV1 knockout and capsaicin pretreated mice. The European journal of neuroscience, 2004. 19(5): p. 1421-4. 
[88] Garami, A., et al., Thermoregulatory phenotype of the Trpv1 knockout mouse: thermoeffector dysbalance with hyperkinesis. The Journal of neuroscience : the official journal of the Society for Neuroscience, 2011. 31(5): p. 1721-33.

[89] Caterina, M.J., et al., A capsaicin-receptor homologue with a high threshold for noxious heat. Nature, 1999. 398(6726): p. 436-41.

[90] Shibasaki, K., et al., TRPV2 enhances axon outgrowth through its activation by membrane stretch in developing sensory and motor neurons. The Journal of neuroscience : the official journal of the Society for Neuroscience, 2010. 30(13): p. 4601-12.

[91] Park, U., et al., TRP vanilloid 2 knock-out mice are susceptible to perinatal lethality but display normal thermal and mechanical nociception. The Journal of neuroscience : the official journal of the Society for Neuroscience, 2011. 31(32): p. 11425-36.

[92] Link, T.M., et al., TRPV2 has a pivotal role in macrophage particle binding and phagocytosis. Nature immunology, 2010. 11(3): p. 232-9.

[93] $\mathrm{Xu}, \mathrm{H}$. , et al., TRPV3 is a calcium-permeable temperature-sensitive cation channel. Nature, 2002. 418(6894): p. 181-6.

[94] Xu, H., et al., Oregano, thyme and clove-derived flavors and skin sensitizers activate specific TRP channels. Nature neuroscience, 2006. 9(5): p. 628-35.

[95] Moqrich, A., et al., Impaired thermosensation in mice lacking TRPV3, a heat and camphor sensor in the skin. Science, 2005. 307(5714): p. 1468-72.

[96] Mandadi, S., et al., TRPV3 in keratinocytes transmits temperature information to sensory neurons via ATP. Pflugers Archiv : European journal of physiology, 2009. 458(6): p. 1093102.

[97] Asakawa, M., et al., Association of a mutation in TRPV3 with defective hair growth in rodents. The Journal of investigative dermatology, 2006. 126(12): p. 2664-72.

[98] Xiao, R., et al., The TRPV3 mutation associated with the hairless phenotype in rodents is constitutively active. Cell Calcium, 2008. 43(4): p. 334-43.

[99] Cheng, X., et al., TRP channel regulates EGFR signaling in hair morphogenesis and skin barrier formation. Cell, 2010. 141(2): p. 331-43.

[100] Liedtke, W., et al., Vanilloid receptor-related osmotically activated channel (VR-OAC), a candidate vertebrate osmoreceptor. Cell, 2000. 103(3): p. 525-35.

[101] Strotmann, R., et al., OTRPC4, a nonselective cation channel that confers sensitivity to extracellular osmolarity. Nature cell biology, 2000. 2(10): p. 695-702.

[102] Chung, M.K., et al., TRPV3 and TRPV4 mediate warmth-evoked currents in primary mouse keratinocytes. The Journal of biological chemistry, 2004. 279(20): p. 21569-75.

[103] Guler, A.D., et al., Heat-evoked activation of the ion channel, TRPV4. The Journal of neuroscience : the official journal of the Society for Neuroscience, 2002. 22(15): p. 6408-14.

[104] Suzuki, M., et al., Impaired pressure sensation in mice lacking TRPV4. The Journal of biological chemistry, 2003. 278(25): p. 22664-8.

[105] Lee, H., et al., Altered thermal selection behavior in mice lacking transient receptor potential vanilloid 4. The Journal of neuroscience : the official journal of the Society for Neuroscience, 2005. 25(5): p. 1304-10. 
[106] Todaka, H., et al., Warm temperature-sensitive transient receptor potential vanilloid 4 (TRPV4) plays an essential role in thermal hyperalgesia. The Journal of biological chemistry, 2004. 279(34): p. 35133-8.

[107] [107] Patapoutian, A., et al., ThermoTRP channels and beyond: mechanisms of temperature sensation. Nature reviews. Neuroscience, 2003. 4(7): p. 529-39.

[108] [108] Hille, B., Ion Channels of Excitable Membranes. 3rd ed. 2001, Sunderland, MA, USA: Sinauer Associates Inc. 